Relations industrielles

Industrial Relations

\title{
Lewandowski, Henryk et Zbigniew Hajn, dir., Le syndicalisme contemporain et son avenir
}

\section{Jacques Desmarais}

Volume 52, numéro 1, 1997

URI : https://id.erudit.org/iderudit/051162ar

DOI : https://doi.org/10.7202/051162ar

Aller au sommaire du numéro

Éditeur(s)

Département des relations industrielles de l'Université Laval

ISSN

0034-379X (imprimé)

1703-8138 (numérique)

Découvrir la revue

Citer ce compte rendu

Desmarais, J. (1997). Compte rendu de [Lewandowski, Henryk et Zbigniew

Hajn, dir., Le syndicalisme contemporain et son avenir]. Relations industrielles /

Industrial Relations, 52(1), 220-223. https://doi.org/10.7202/051162ar

Tous droits réservés (C Département des relations industrielles de l'Universite Laval, 1997
Ce document est protégé par la loi sur le droit d'auteur. L'utilisation des services d'Érudit (y compris la reproduction) est assujettie à sa politique d'utilisation que vous pouvez consulter en ligne.

https://apropos.erudit.org/fr/usagers/politique-dutilisation/ 
système ferroviaire. À ce niveau, à la lumière des études récentes menées, notamment en ergonomie, sur le travail des équipes de train, les trente ans passés ne semblent pas avoir rendu obsolètes les observations de Morgan. Sa lecture du pouvoir informel détenu par les équipes de train est, trente ans plus tard, toujours actuelle. D'autre part, cet ouvrage intéressera ceux qui, notamment en sociologie, ergonomie et psychodynamique du travail, sont concernés par l'étude des pratiques informelles et du contrōle des travailleurs sur l'organisation de leur propre travail. Pool et Young ont eu une excellente idée en rééditant l'ouvrage de Morgan. Leur introduction permet une meilleure connaissance de l'auteur de l'ouvrage original et des contextes (social, économique et politique) dans lesquels l'industrie ferroviaire évoluait dans les années 60. L'ajout de notes en bas de page ne fait, quant à lui, que rendre le document conforme aux règles de rédaction actuelles.

\section{NANCY CAMERON FERNANDE LAMONDE Université Laval}

\section{Le syndicalisme contemporain et son avenir / Colloque international orga- nisé par la Chaire de droit du travail et de la sécurité sociale de l'Univer- sité de Lodz \\ édité par Henryk LEWANDOWSKI et Zbigniew HAJN, Lódz: Wydawnictwo Uniwerytetu Lódzkiego, 1995, 276 p., ISBN 83-7016-958-9.}

Cet ouvrage est le compte rendu d'un échange entre des spécialistes du droit du travail invités à l'Université de Lódz, Pologne, en octobre 1993, pour y présenter le portrait de l'état du syndicalisme de leur pays et leur évaluation sur son avenir. Présenter l'état des lieux syndicaux de cinq pays de l'Union européenne (France, Allemagne, Italie, Grande-Bretagne et Espagne), sept de l'Europe centrale et orientale (Pologne, Hongrie, République tchèque, Slovaquie, Roumanie, Bulgarie et Russie) ainsi que du Canada, suscite dès l'abord plusieurs questions. Comment se comportent des systèmes de relations professionnelles aussi divers aux conjonctures globale et régionale ? Comment réagit-on à la restructuration du marché du travail et à la croissance des exclus sociaux et qu'arrive-t-il à la représentativité des syndicats, surtout intéressés par la représentation des travailleurs qui ont un emploi ? Comment vit-on l'intégration à l'Union européenne et quel est l'effet du passage à l'économie de marché chez les "nouveaux "syndicats des pays de l'Europe centrale et orientale qui ont contribué au mouvement de libéralisation? Qu'en estil de la concurrence induite par la mondialisation des échanges? L'offensive du patronat des pays développés braqué sur le contrôle des coûts de la main-d'œuvre et assidûment en demande pour "plus de flexibilité " dans les règles du travail et ce, dans chaque établissement selon ses propres besoins de production, favorise-t-il vraiment un mouvement de décentralisation des négociations? Ces nouvelles conditions et d'autres plus dépendantes de l'évolution historique de chaque pays commandent une orientation diverse chez les uns et les autres. Voyons schématiquement ce que cet ouvrage nous montre au sujet des pays exposés.

\section{L'Europe occidentale}

En France, où le déclin de la syndicalisation est manifeste et où la recomposition syndicale semble non envisageable, l'action syndicale doit être menée sur trois fronts: (1) les négociations collectives à l'échelle européenne et avec des groupes multinationaux en 
fréquente restructuration; (2) la négociation d'accords nationaux sur l'emploi et le partage du travail pour faire reculer la dualisation et l'exclusion; enfin, (3) tendance préoccupante pour la coexistence du pluralisme et de l'égalité, la négociation d'accords d'entreprise avec comme enjeu l'exercice efficace du droit syndical dans l'entreprise (financement patronal, protection de la carrière des salariés exerçant des mandats représentatifs, nouvelles instances de représentation) et la régression des conditions de travail par rapport aux conventions de branche.

En Allemagne, la réunification avec l'Allemagne de l'Est et l'inévitable rattrapage progressif des conditions de travail de ces salariés ont mobilisé l'action syndicale. Ensuite, orientation plus lourde, l'offensive patronale pour " plus de flexibilité " a favorisé la négociation d'accords par établissement où les conseils d'établissement représentatifs de l'ensemble des salariés se sont avérés des partisans naturels des intérêts locaux, parfois au détriment des visions macroéconomiques des syndicats sectoriels. Ces confrontations continueront et la volonté syndicale de protéger la politique sociale allemande sera mise à rude épreuve, même avec une représentativité en hausse (de 30 à $38 \%$ depuis la réunification).

En Italie, la représentativité syndicale, confrontée aux nouvelles formes du travail précaire et aux responsabilités du partage des sacrifices induits par le contexte économique, est tiraillée entre l'action centralisée, la négociation d'une politique des revenus, et celle, traditionnelle, sur les lieux de travail. De plus, toujours encadrée par la convention de branche, la convention collective d'entreprise investissant le champ de la politique de l'emploi est maintenant appelée à contrōler les nouvelles formes d'emploi et à décider de l'emploi intérimaire dans l'entreprise. L'agenda syndical est tout tracé : comment aménager les règles des nouvelles formes d'emploi et contrôler leur application devant la ten- tation légendaire de l'employeur de "fuir le droit ".

En Espagne, l'ère post-franquiste, tout en favorisant la liberté syndicale, a maintenu les comités d'entreprises composés de représentants des travailleurs élus avec l'accord des syndicats "suffisamment" représentatifs et composés de candidats provenant des syndicats "légalement" constitués, sans édicter toutefois le droit des travailleurs à la gestion de l'entreprise. Or, malgré un faible taux d'affiliation (env. $5 \%$ ), cette dualité structurelle encourage un affrontement continu entre des bureaucraties surtout politiques (socialistes et communistes), la cause d'un avenir que l'auteur juge avec pessimisme, tant que continuera la main-mise de ces deux syndicats sur la vie syndicale.

En Grande-Bretagne, le portrait détaillé de l'ensemble des mesures adoptées par le gouvernement conservateur depuis le début des années 80 pour restreindre l'action syndicale a entraîné un affaiblissement généralisé. L'avenir des syndicats, dont les pouvoirs autonomes sont passés sous un contrôle étatique croissant, laisse peu d'espoir - du moins à l'automne 1993 - qu'un éventuel gouvernement travailliste ravive la situation d'antan.

\section{Le Canada}

Au Canada, où, à la différence des États-Unis, la représentativitê syndicale et les conditions d'exercice de l'action syndicale demeurent relativement favorables, le contexte de l'action syndicale a été modifié par la globalisation des marchés, la mutation des structures de l'emploi et du marché du travail, l'extériorisation de la production et la croissance du travail autonome. Le système de représentation syndicale conçu pour le travail subordonné et axé principalement sur la convention collective d'entreprise semble stable, tout limité qu'il soit en tant que mode de participation des salariés. D'autre part, l'évolution de ce contexte devrait 
favoriser un déplacement du lieu de décision, de l'entreprise vers le secteur, plus apte à permettre les concertations utiles, sans besoin de modifier le modèle nordaméricain d'exclusivité de représentation adaptable à une telle évolution. Enfin, ces nouveaux enjeux sont susceptibles d'entraîner progressivement une extension de l'implication sociopolitique des syndicats.

\section{L'Europe centrale et orientale}

La présentation du nouveau mouvement syndical dans les pays de l'Europe centrale et orientale (Pologne, Hongrie, République tchèque, Slovaquie, Roumanie, Bulgarie et Russie) constitue la pièce de résistance de l'ouvrage. L'ensemble des nouvelles structures syndicales de chacun des pays est présenté ainsi que les conditions juridiques et institutionnelles de cette rénovation syndicale.

Le droit positif a été transformé. La constitutionnalisation de la liberté d'association a dorénavant une valeur normative et la législation du travail est progressivement mise à niveau avec le droit international du travail. La convention collective de travail est l'instrument privilégié de l'action syndicale, mais sans exclusive : à défaut de syndicat, d'autres organes mandatés par les salariés ou des représentants élus du personnel peuvent y participer. Les objets possibles de la convention collective semblent laissés à l'initiative des parties, mais en respectant la suprématie de la loi sur la convention collective. La participation syndicale à la gestion des entreprises n'est reconnue que de façon consultative ; toutefois, on a décrété l'obligation d'information sur des questions importantes ou qui concernent la majorité des salariés, une disposition cruciale en cette période de licenciements collectifs nombreux.

La grève est réglementée: son exercice est restreint dans les services publics et des étapes préalables de "règlement pacifique" sont prévues; parfois autorisée en seule période de négociation collective, la grève ne peut porter que sur la revendication de conditions de travail et non sur l'application des droits reconnus par convention collective. La grève "sauvage " est tolérée et, à ce jour, le lock-out demeure interdit.

À l'échelle nationale, le tripartisme est favorisé. La négociation d'accords généraux sur des éléments de réforme économique, comme l'emploi, les prestations sociales et les salaires est devenue l'autre voie d'une action syndicale parsemée d'obstacles : la faiblesse de la participation du patronat du secteur privé rend ce tripartisme factice; l'action syndicale est surtout efficace au niveau local. Enfin, la pluralité des organisations syndicales rendent précaires ces accords nationaux, d'autant plus que les gouvernements eux-mêmes éprouvent des difficultés à faire adopter par les parlements les lois consécutives à ces accords.

Comment ont réagi les syndicats au mouvement de libéralisation? La libéralisation politique n'a pas entraîné la dépolitisation des syndicats, mais a favorisé une pluralité de modes d'action : l'intensité et la forme de l'action politique syndicale varient de la tendance corporatiste, à l'appui circonstanciel ou permanent aux formations politiques jusqu'à la participation directe du syndicat au sein du Parlement. Les réformes économiques ont entraîné des bouleversements majeurs. Initialement appuyé par les syndicats, le mouvement s'est heurté à la résistance syndicale lorsque ses coûts sociaux ont commencé à heurter de front les salariés et les retraités. La baisse des prestations sociales et le chômage croissant ont mobilisé les syndicats en fait incapables de résister aux conséquences de la libéralisation et surtout de la privatisation de l'économie. Une opposition entre la direction et la base syndicale a dès lors surgi, et plus vivement lorsque la participation de l'élite syndicale était liée à l'action gouvernementale. D'où le débat typique, notamment en Pologne avec Solidarnosc, au sujet du rôle respectif du 
syndicat et du parti, entre les fonctions de revendication et de coopération.

Quel avenir voit-on se dessiner? Les directions politiques craignent de plus en plus l'action politique des syndicats, d'où une réaction à prévoir de leur part en vue de la contrôler. Les syndicats continueront d'opposer une vive résistance aux attaques contre les régimes de sécurité sociale. La pratique de négociation des accords généraux devrait croître. Les exigences de l'action de revendication syndicale devraient rapprocher et consolider les syndicats au delà des divergences idéologiques. Un mouvement de consolidation des petites structures syndicales devrait voir le jour. L'expansion du secteur privé ne sera pas accompagné d'un mouvement équivalent de syndicalisation, la nouvelle structure du marché du travail vers la tertiarisation constituant un frein au maintien soutenu de la présence syndicale.

\section{Conclusion}

Cet ouvrage est riche d'informations sur l'ampleur et la diversité des défis lancés aux syndicats dans la conjoncture actuelle. Sans recourir au diagnostic alarmiste d'une "crise " syndicale, le portrait de chaque pays révèle cependant un malaise généralisé et multiforme: la représentativité syndicale est affaiblie en même temps que croissent le chômage et le travail non salarié; le pluralisme encourage la division; le dualisme des modes de représentation amène des confrontations entre des intérêts locaux au détriment de visions élargies ; la sécurité et l'universalité de la convention collective sont ébranlées par les accords atypiques décentralisés; la concertation et la participation sont valorisées de préférence à la confrontation et à la revendication; le tripartisme à l'échelle nationale porte à la coopération et mine la légitimité de l'action syndicale de revendication. L'action syndicale subit en fait une mutation suscitée par la complexité des contraintes créées par la nouvelle diversité des statuts du travail subordonné et par les modes éclatés de production d'une entreprise de moins en moins durable et fixe, de plus en plus provisoire et mobile, voire virtuelle. Comment en fait agir à la fois sur le global et le local, comment continuer de revendiquer tout en voulant coopérer?

L'ouvrage ne propose pas de réponse globale toute faite, mais il a le mérite de poser les questions cruciales et de contribuer au questionnement sur l'avenir des syndicats. Cependant, et à regret devant l'effort consenti pour la seule publication de l'ouvrage, nous devons signaler la faible qualité de la traduction de plusieurs textes au point où parfois le sens de certains passages devient difficile à saisir. Mis à part ce désagrément, l'ouvrage constitue un bon outil de référence pour son vaste panorama de l'action syndicale et pour la franchise de ses auteurs dans leur analyse des enjeux contemporains.

JACQUES DESMARAIS

Université du Québec à Montréal

\section{Seniority and Employment Equity for Women}

par Louise DULUDE, Kingston, Ont. : Queen's University at Kingston, Industrial Relations Center, 1995, 154 p., ISBN 0-88886-395-0.

Dans cet ouvrage, l'auteure explore certains effets discriminants des dispositions des conventions collectives relatives à l'ancienneté sur les femmes et sur les mesures d'accès à l'égalité, lorsqu'il y en a. Elle se propose de faire l'état des moyens que peuvent utiliser les femmes pour lutter contre ces effets, dans l'état actuel du droit. Elle le fait sans par ailleurs s'en prendre à l'existence du principe fondamental de l'ancienneté, dont elle ne souhaite pas tant l'élimination que 